\title{
NEW METHODS IN COINCIDENCE THEORY
}

\author{
KALYAN K. MUKHERJEA ${ }^{1}$
}

\begin{abstract}
Cobordism theory is used to obtain a new type of result concerning the coincidence of maps between compact manifolds.
\end{abstract}

1. Introduction. Coincidence theory concerns itself with the following basic question: Given two maps $f, g: X \rightarrow Y$ of topological spaces, when is there a coincidence point, i.e. a point $x \in X$ such that $f(x)=g(x)$ ? In this generality one can say almost nothing of interest, so we concern ourselves with the case when $X$ and $Y$ are compact manifolds. When $\operatorname{dim} X=\operatorname{dim} Y$, and both the manifolds are oriented, we have the famous Lefschetz coincidence theorem which associates a number-the Lefschetz number-to the pair of maps $f$ and $g$ and if this number is nonzero, there is a coincidence of $f$ and $g$. See [7]. Both the formulation of the Lefschetz number and the proof of the theorem is similar to Lefschetz's original proof of the fixed-point theorem for compact manifolds.

If $\operatorname{dim} X<\operatorname{dim} Y$, a simple general-position or transversality type argument shows that the maps $f$ and $g$ may be homotopically deformed to be coincidence-free and hence there can be no coincidence theorem in the realm of the homotopy category or algebraic topology.

The case $\operatorname{dim} X>\operatorname{dim} Y$ is more interesting and Lefschet $z$ tackled this case, though without much success. Indeed Steenrod has suggested [7, p. 28] that it was this problem which partially motivated Lefschetz in his studies of the cup- and cap-products. It is a bit surprising that in spite of the basic nature of the question and the above interesting historical associations, coincidence theory has been almost totally neglected and in fact modulo some technical refinements, Lefschetz's coincidence theorem is the only result in coincidence theory.

Presented to the Society, March 26, 1971; received by the editors October 7, 1971. AMS 1970 subject classifications. Primary 55C20; Secondary 55B20.

Key words and phrases. Coincidence, cobordism, $K$-theory.

${ }^{1}$ Research was partially supported by National Science Foundation grant GP-27276. Much of the work presented was done during the author's sabbattical visit to the Tata Institute of Fundamental Research, Bombay. The author takes this opportunity to thank the members of the School of Mathematics, T.I.F.R. for their kindness and hospitality.

(c) American Mathematical Society 1972 
In this note $\mathrm{I}$ indicate how cobordism and $K$-theory may be used to attack the coincidence problem, obtain a general coincidence class which lives in a certain cobordism group and indicate how this invariant can be used in applications. Further refinements and applications will be published later.

2. Cobordism and coincidence. We shall work throughout in the Diff $^{\infty}$ category, though the main result and its proof may be adapted to the categories PL and TOP. The cobordism theory used is very elementary; for a simple and extremely elegant geometrical treatment, see [6].

Let $G$ be either the stable orthogonal group $\boldsymbol{O}$, stable special orthogonal group, $\boldsymbol{S O}$, or stable unitary group $\boldsymbol{U}$. Corresponding to these we get bordism theories, $\Omega_{*}^{G}$, of unoriented, oriented and weakly almost complex manifolds and the corresponding cobordism theories, $\Omega_{G}^{*}$.

Suppose $X, Y$ are compact manifolds whose stable tangent bundles have a $G$-reduction, $f, g: X \rightarrow Y$ two maps and $\operatorname{dim} X=m, \operatorname{dim} Y=n$, $m \geqq n$.

Suppose $f$ and $g$ are transverse regular. Then the coincidence set

$$
C_{f, g}=\{x \in X \mid f(x)=g(x)\} \subset X
$$

is an $(m-n)$-dimensional closed submanifold and its normal bundle $v_{f, g}$ in $X$ is the pullback of the normal bundle $v_{\Delta}$ of the diagonal $\Delta \subset Y \times Y$ under the map

$$
\begin{aligned}
f \times g: X & \rightarrow Y \times Y, \\
x & \mapsto(f(x), g(x)) .
\end{aligned}
$$

Thus $C_{f, g} \subset X$ gives rise to a class $L_{f, g} \in \Omega_{G}^{n}(X)$, which is the obvious one if one chooses Quillen's formulation or the Atiyah dual of the coincidence submanifold if one prefers the abstract formulation.

Since $L_{f, g}=L_{f^{\prime}, g^{\prime}}$ if $f$ and $f^{\prime}$ and $g$ and $g^{\prime}$ are homotopic, we immediately get:

TheOREm. Let $X, Y$ be as above and let $f, g: X \rightarrow Y$ be continuous maps. Define

$$
L_{f, g}=(f \times g)^{*}\left(\left(\Delta_{Y}\right)_{!}(1)\right)
$$

where $1 \in \Omega_{G}^{0}(Y)$ and $\left(\Delta_{Y}\right)_{!}: \Omega_{G}^{*}(Y) \rightarrow \Omega_{G}^{*+n}(Y \times Y)$ is the "umkehrungshomomorphismus" or direct image map [3] induced by the diagonal map, $\Delta_{Y}: Y \rightarrow Y \times Y$. If $L_{f, g} \neq 0, f$ and $g$ have a coincidence.

Proof. First note that $\left(\Delta_{Y}\right)_{!}(1)$ is just an alternative way of denoting the cobordism class of the diagonal imbedding of $Y \rightarrow Y \times Y$ and thus if $f$ and $g$ are transversal $C^{\infty}$ maps, $L_{f, g}$ as defined in $\left(^{*}\right)$ is the same as the 
previous definition. Now a standard approximation argument yields the result.

Caution. We have been abusing language a great deal by not specifying the "orientations" of the imbeddings $C_{f, g} \rightarrow X$ and $Y \rightarrow Y \times Y$; we of course choose the standard ones arising from the natural isomorphism $v_{\Delta} \cong \tau_{Y}$, ( $\tau$ 三tangent bundle).

REMARKS. (1) When $m=n$, the coincidences are isolated points, in the generic case. The Lefschetz number is the algebraic number (when $G=$ $\boldsymbol{S O}, \boldsymbol{U}$ ) or the parity (when $G=\boldsymbol{O}$ ) of coincidence points. Thus the use of cobordism is superfluous; rational or $Z_{2}$-cohomology suffices. In coincidence theory however, one cannot disregard torsion. We give an example below to show how the finer torsion present in the $K$-theory of lens spaces (finer than integral cohomology, that is) can be used to detect coincidences that are otherwise invisible.

(2) What we have described is really a special case of the following general formalism: Let $Y$ be any differentiable manifold and $Y^{\tau}$ the Thom space of its tangent bundle. Let $h^{*}$ be any cohomology theory and $f, g: X \rightarrow Y$ two morphisms in the category on which $h^{*}$ is defined. If there is an $\alpha \in h^{*}\left(Y^{r}\right)$ such that the image of $\alpha$ under the map induced by

$$
X \stackrel{f \times g}{\longrightarrow} X \times Y \stackrel{p}{\longrightarrow} Y^{r}
$$

where $p$ is the Pontryagin-Thom collapsing map, is nonzero, then $f$ and $g$ have coincidences.

(3) Also in the present context, we can replace compactness of $X$ and $Y$ by using cobordism with supports [5] and require that one map be proper and the other one compact.

3. On using the theorem. The theorem we proved in the last section is not, as it stands, in a particularly useful form since $L_{f, g}$ is all but impossible to compute. To use it for applications, tricks must be found according to the situation in which one is interested. We mention two methods which apply for the weakly-complex case.

There is a multiplicative transformation [2], $\mu_{C}: \Omega_{U}^{*} \rightarrow H^{* *}(; Q)$, which may in fact be factored:

$$
\Omega_{U}^{*} \stackrel{\mu}{\longrightarrow} \tilde{K}^{*} \stackrel{\mathrm{ch}}{\longrightarrow} H^{* *}(; Q)
$$

where ch is the Chern character and $\mu(\alpha)=\alpha^{*}(\beta)$ where $\alpha$ is now looked upon as a homotopy class of maps into $M U$, the Thom spectrum, and $\beta$ the virtual bundle on $M U(n)$ for large $n$, given by Bott periodicity.

The Riemann-Roch theorem [3] for the transformation $\mu_{c}$, yields:

$$
\mu_{c}\left(L_{f, g}\right)=(f \times g)^{*}\left(\Delta_{Y}\right)_{*}(\operatorname{td} Y)^{-1}
$$


where $\left(\Delta_{Y}\right)_{*}$ is the Gysin homomorphism in rational cohomology and ( $\operatorname{td} Y$ ) is the Todd genus of $\tau_{Y}$, regarded as a complex vector bundle. Considering the map $X \rightarrow \mathrm{pt}$, one gets again by Riemann-Roch:

$$
\left\langle(f \times g)^{*} \Delta_{*}(\operatorname{td} Y)^{-1} \cdot(\operatorname{td} X),[X]\right\rangle=\left\langle\operatorname{td} C_{f, g},\left[C_{f, g}\right]\right\rangle .
$$

By suitable manipulations one can determine all the $K$-theory characteristic numbers of $C_{f, g} \subset X$ and this determines its bordism class if $X$ has no torsion in its integral homology.

If the $K$-theory of $X$ and $Y$ are known and $Y$ is torsion free, one can use $\mu: \Omega_{U}^{*} \rightarrow \widetilde{K}^{*}$ and work with $K$-theory. One has

$$
\mu\left(\Delta_{Y}\right)_{!}(1)=\left(\Delta_{Y}^{K}\right)_{!}(1)
$$

where $\left(\Delta_{Y}^{K}\right)$ i is the direct image in $K$-theory. Now using the RiemannRoch theorem for ch: $\widetilde{K}^{*} \rightarrow \widetilde{H}^{* *}(; Q)$ one can (in principle) compute $\left(\Delta_{Y}^{K}\right)_{i}(1) \in \widetilde{K}^{0}(Y \times Y)$ as a sum of tensor-products of virtual bundles. (Note, that by the Künneth formula [1], $K(Y \times Y) \cong K(Y) \otimes K(Y)$ since the Tor vanishes.)

For example, if $Y=C P_{n}, h$ the Hopf line bundle on $C P_{n}$ and $\xi=$ $[h-1] \in \tilde{K}^{0}\left(C P_{n}\right)$, we have

Proposition.

$$
\left(\Delta_{Y}^{K}\right)_{!}(1)=\sum_{k=0}^{n} t_{k} \sum_{i+j=n+k ; 0 \leqq i, j \leqq n} \xi^{i} \bowtie \xi^{j}
$$

where $t_{k}$ is the coefficient of $x^{k}$ in the power series expansion of $(1+x)^{-n}$ and $\otimes$ is the external tensor product.

Now knowing $\left(\Delta_{Y}^{K}\right)_{!}(1)$, we have $\mu\left(L_{f, g}\right)=(f \times g)^{*}\left(\Delta_{Y}^{K}\right)_{!}(1)$ and the nonvanishing of $\mu\left(L_{f, g}\right)$ implies the existence of coincidences. We give an illustration of this technique.

EXAMPLE. Let $p$ be any odd prime. Then, $S^{4 p-1} \times S^{4 p-1}$ has a standard complex structure $\boldsymbol{Z}_{p} \times \boldsymbol{Z}_{p}$ acts on this in holomorphic manner, without fixed points; the generators acting as follows:

$$
\begin{aligned}
& \left(Z_{0}, \cdots, Z_{2 p-1}, W_{0} \cdots W_{2 p-1}\right) \leadsto\left(Z_{0} \cdot \zeta, \cdots, Z_{2 p-1} \cdot \zeta, W_{0} \cdots W_{2 p-1}\right), \\
& \left(Z_{0}, \cdots, Z_{2 p-1}, W_{0} \cdots W_{2 p-1}\right) \leadsto\left(Z_{0} \cdots Z_{2 p-1}, W_{0} \cdot \zeta \cdots W_{2 p-1} \cdot \zeta\right)
\end{aligned}
$$

where $\zeta=e^{2 \pi i / p}$. The quotient space is a product of lens spaces,

$$
L^{2(p-1)}(p) \times L^{2(p-1)}(p),
$$

and is a complex manifold.

The $K$-theory of $L^{2(p-1)}(p)$ is described as follows [4]:

Let $\pi: L^{2(p-1)}(p) \rightarrow C P_{2(p-1)}$ be the natural projection and $t \rightarrow L^{2(p-1)}(p)$ the pullback of the Hopf bundle under $\pi$. Let $\sigma=\left[t-1_{C}\right] \in \widetilde{K}^{0}\left(L^{2(p-1)}(p)\right)$. 
Then,

and

$$
\tilde{K}^{0}\left(L^{2(p-1)}(p)\right)=Z_{p^{2}}[\sigma]+Z_{p^{2}}\left[\sigma^{2}\right]+\cdots+Z_{p^{2}}\left[\sigma^{p-1}\right]
$$

$$
\sigma^{p}=-\sum_{i=1}^{p-1}\left(\begin{array}{l}
p \\
i
\end{array}\right) \sigma^{p-i}, \quad \sigma^{2 p-1}=0 .
$$

Let $\quad X=L^{2(p-1)}(p) \times L^{2(p-1)}(p) \times C P_{p-1}, \quad Y=C P_{2(p-1)} \times C P_{p-1}$. The $K-$ theories of $X$ and $Y$ can be described by means of the Künneth formula.

Let $f, g: X \rightarrow Y$ be any two maps homotopic to

$$
\begin{aligned}
& \left(\pi \cdot \pi_{1}\right) \times \mathrm{id}: L^{2(p-1)}(p) \times L^{2(p-1)}(p) \times C P_{p-1} \rightarrow C P_{2(p-1)} \times C P_{p-1}, \\
& \left(\pi \cdot \pi_{2}\right) \times \mathrm{id}: L^{2(p-1)}(p) \times L^{2(p-1)}(p) \times C P_{p-1} \rightarrow C P_{2(p-1)} \times C P_{p-1}
\end{aligned}
$$

where $\pi_{1}, \pi_{2}$ are the projections on the first and second factors of $X$ respectively.

A simple calculation shows that the homology class of the coincidence set is zero. But, $\mu\left(L_{f, g}\right)=p \cdot \sigma_{1}^{p-1} \otimes \sigma_{2}^{p-1} \otimes \xi^{p-1}$ where $\sigma_{1}=\pi_{1}^{*}(\sigma), \sigma_{2}=\pi_{2}^{*}(\sigma)$ and $\xi=\pi_{3}^{*}(\xi), \xi$ the Hopf bundle on $C P_{p-1}$. (See Appendix below.)

The Künneth formula implies that $\sigma_{1}^{p-1} \otimes \sigma_{2}^{p-1} \otimes \xi^{p-1}$ has order $p^{2}$ and hence $\mu\left(L_{f, g}\right) \neq 0$.

Thus $f$ and $g$ have coincidences even though they cannot be detected by means of ordinary cohomology (any coefficients!).

Appendix. Note, first of all, that $f$ and $g$ are obtained by taking the maps

$$
\begin{aligned}
& \pi \cdot \pi_{1}: L^{2(p-1)}(p) \times L^{2(p-1)}(p) \rightarrow C P_{2(p-1)}, \\
& \pi \cdot \pi_{2}: L^{2(p-1)}(p) \times L^{2(p-1)}(p) \rightarrow C P_{2(p-1)}
\end{aligned}
$$

and crossing each with id: $C P_{p-1} \rightarrow C P_{p-1}$. The Lefschetz number $L$ (id, id $)=p$ and thus the effect on the coincidence submanifold is to "multiply it by $p$ ", i.e.

$$
\mu\left(L_{f, g}\right)=\left(p \cdot \xi^{p-1}\right) \cdot \mu\left(L_{\pi \cdot \pi_{1}, \pi \cdot \pi_{2}}\right) .
$$

These remarks apply in cohomology as well. Since the Poincaré dual of the coincidence submanifold in $(* *)$ has order $p$, the coincidence submanifold of $f$ and $g$ has zero (homology) fundamental class.

Now for the $K$-theory computation: Note, that $\sigma^{p}=-\sum_{i=1}^{p-1}\left(\begin{array}{l}p \\ i\end{array}\right) \sigma^{p-i}$ and $\operatorname{ord}\left(\sigma^{j}\right)=p^{2}$ for $1 \leqq j \leqq p-1$ implies $\sigma^{k}$ has order at most $p$ if $k>p-1$ ( $p$ is prime).

Now,

$$
\mu\left(L_{f, g}\right)=\mu\left(L_{\pi \cdot \pi_{1}, \pi \cdot \pi_{2}}\right) \otimes p \xi^{p-1}
$$


and

$$
\mu\left(L_{\pi \cdot \pi_{1}, \pi \cdot \pi_{2}}\right)=\sum_{k=0}^{2(p-1)} t_{k} \sum_{i+j=2(p-1)+k} \sigma_{1}^{i} \otimes \sigma_{2}^{j} .
$$

Every element in this sum has order at most $p$, except $\sigma_{1}^{p-1} \otimes \sigma_{2}^{p-1}$. Hence $\mu\left(L_{f, g}\right)=p \cdot \sigma_{1}^{p-1} \otimes \xi^{p-1}$.

\section{REFERENCES}

1. M. F. Atiyah, $K$-theory, Benjamin, New York, 1967. MR 36 \#7130.

2. P. E. Conner and E. E. Floyd, The relation of cobordism to $K$-theories, Lecture Notes in Math., no. 28, Springer-Verlag, Berlin and New York, 1966. MR 35 \#7344.

3. E. Dyer, Cohomology theories, Math. Lecture Note Series, Benjamin, New York, 1969. MR 42 \#3780.

4. T. Kambe, The structure of $K_{\Lambda^{-}}$-rings of the Lens space and their applications, J. Math. Soc. Japan 18 (1966), 135-146. MR 33 \#6646.

5. J. J. Morava, Fredholm maps and Gysin homomorphisms, Proc. Sympos. Pure Math., vol. 15, Amer. Math. Soc., Providence, R.I., 1970, pp. 135-156. MR 42 \#3817.

6. D. Quillen, Elementary proofs of some results of cobordism theory using Steenrod operations, Advances in Math. 7 (1971), 29-56.

7. N. E. Steenrod, The work and influence of Professor S. Lefschetz in algebraic topology, Algebraic Geometry and Topology (Sympos. in Honor of S. Lefschetz), Princeton Univ. Press, Princeton, N.J., 1957, pp. 24-43. MR 19, 158.

Department of Mathematics, University of California, los Angeles, CaliFORNIA 90024 